\title{
A Healthy Eating Education Program for Midwives to Investigate and Explore Their Knowledge, Understanding, and Confidence to Support Pregnant Women to Eat Healthily: Protocol for a Mixed-Methods Study
}

Shwikar Mahmoud Etman Othman ${ }^{1,2}$, RN, BNSc, MD; Mary P Steen², RGN, RM, DipClinHypn, PGCRM, PGDipHE, MCGI, PhD; Rasika Jayasekara², RN, BA, BScN (Hons), PGDipEdu, MNSc, MClinSc, PhD; Julie-Anne Fleet ${ }^{2}$, BAappSc, Grad Cert, BMid (Hons), PhD

${ }^{1}$ Obstetrics and Gynaecology Nursing Department, Faculty of Nursing, South Valley University, Qena, Egypt

${ }^{2}$ School of Nursing and Midwifery, Division of Health Sciences, University of South Australia, Adelaide, Australia

\section{Corresponding Author:}

Shwikar Mahmoud Etman Othman, RN, BNSc, MD

School of Nursing and Midwifery

Division of Health Sciences

University of South Australia

GPO Box 2471, Adelaide SA 5001

Adelaide,

Australia

Phone: 610470626813

Email: othsm001@mymail.unisa.edu.au

\begin{abstract}
Background: Nutrition and healthy eating behaviors during pregnancy are vitally important for the health of a mother and her developing baby. However, some midwives have reported a lack of evidence-based nutrition knowledge for providing information about healthy eating to women during pregnancy.

Objective: In this study, the aim is to design and evaluate a healthy eating education program to enhance midwives' knowledge, understanding, and confidence to support pregnant women in South Australia to make healthy eating choices.

Methods: This mixed-methods study consists of two phases. The first phase, Phase 1, consists of an education program for midwives, "Healthy Eating in Pregnancy," to be delivered through a workshop or webinar. Each midwife will attend one workshop or webinar, which will be approximately two hours in length. This program will be evaluated through pre-, immediate-, and post-educational questionnaires utilizing a website specifically designed for this study. The participants will be midwives who are members of the Australian College of Midwives and the Australian Nursing and Midwives Federation, and users of social media (eg, Facebook and Twitter) residing and employed in South Australia. Phase 2 will consist of semistructured interviews with a purposive sample of midwives. These interviews will be undertaken to gain an in-depth understanding of midwives' views and how confident they feel educating pregnant women after receiving the healthy eating education. Interviews will be face-to-face or conducted by telephone with midwives who have participated in the healthy eating educational program.
\end{abstract}

Results: A systematic review has previously been undertaken to inform this study protocol. This paper describes and discusses the protocol for this mixed-methods study, which will be completed in April 2019.

Conclusions: The results from the systematic review suggest that there is clear justification to undertake this mixed-methods study to investigate and explore midwives' knowledge, understanding and confidence to support healthy eating in pregnant women. The results and conclusions from the systematic review provided some guidance for the design and development of this study protocol. This mixed-methods study will address a gap in the literature. The results from quantitative and qualitative data sources in this proposed study will help to draw conclusions to address the research topic.

Registered Report Identifier: RR1-10.2196/9861

(JMIR Res Protoc 2018;7(5):e143) doi: 10.2196/resprot.9861 


\section{KEYWORDS}

healthy eating; midwives; education program; mixed-methods research; pregnancy; study protocol

\section{Introduction}

\section{Overview of Influences for a Healthy Pregnancy}

Cumulative evidence demonstrates a pregnant woman's health behaviors have a life-long influence on her health, and that of her developing baby. Good maternal and fetal outcomes have been associated with healthy nutritional habits and an active lifestyle during pregnancy [1,2]. Poor pregnancy outcomes such as preterm birth and small or large for gestational age are often associated with maternal body mass index disorders and life style choices, for example, being underweight or overweight, as well as smoking and alcohol consumption which are modifiable risk factors [3-6]. Unhealthy maternal behavior in pregnancy has been shown to have long-term effects on children and has been associated with conditions such as cognitive defects, obesity, asthma, and cardiovascular diseases [7-11]. Therefore, nutritional education during pregnancy has an important role in maintaining a healthy status for pregnant women.

\section{Health Education During Pregnancy}

Pregnancy is an opportunity to develop or maintain healthy behaviors, as most pregnant women will be motivated to gain more knowledge in order to give their developing fetus the best start in life [12]. Generally, midwives provide health education for pregnant women during antenatal visits. During antenatal visits, some women prefer verbal advice rather than written information from midwives [13]. It is good practice to first verbally discuss and give an explanation about healthy eating in pregnancy, followed by providing written information to help women understand more clearly. Therefore, written material is a complementary source of information [12]. Midwives have an important public health role and provide health education to pregnant women and new mothers.

\section{Midwives' Public Health Role}

National and international maternity policies value and support the role of midwives in public health [14]. Health care providers, such as midwives, have a unique opportunity to support women to make choices to promote health and prevent illness [14]. To achieve this goal the emphasis of midwifery policy and practice is to build relationships with the women they care for and to focus on health promotion as a holistic approach [14]. Therefore, the current study will examine the midwives' role to promote healthy eating, as well as develop and evaluate an educational program for midwives to complement their public health role.

\section{Significance of Nutritional Health Education Programs}

A systematic review is being conducted which aims to examine the effectiveness of healthy eating education programs for improving midwives' level of knowledge and confidence in promoting healthy eating in pregnant women [15]. This review will consider studies that evaluate diet and nutritional education programs, or training targeted for midwives and student midwives (hospital or community-based midwives, obstetric nurses, birth attendants, doulas, midwives alone or with other health professionals) to provide healthy eating knowledge and advice for pregnant women. The education and training program proposed in this mixed-methods study will be a structured program therefore the considered studies in the review may have a defined period, facilitated as a workshop or seminar. The education and training program can be provided by any health care professionals such as dietitians, nutritionists, midwives, health educators, or any other accredited personnel. The primary outcomes of the systematic review are as follows: (1) level of knowledge acquired by midwives and student midwives regarding diet and nutritional requirements in pregnancy measured by any scale or questionnaire and (2) the level of confidence acquired by midwives and student midwives regarding diet and nutritional requirements in pregnancy measured by any scale or questionnaire.

The systematic review was conducted using a three-stage comprehensive search of seven electronic databases and grey literature. Two independent reviewers assessed each paper prior to inclusion using the standardized critical appraisal instruments for evidence of effectiveness developed by the Joanna Briggs Institute. Preliminary results from this systematic review provided guidance for the design of this mixed-methods study and the development of a semistructured questionnaire to be used at the three-time points during the study. The questionnaire will gather data to investigate midwives' level of knowledge and confidence to provide healthy eating education and support to pregnant women. The literature highlighted that there is a lack of studies exploring the role of midwives in antenatal nutritional health education [16-18]. The lack of evidence, therefore, justifies exploring the public health role of midwives to address the nutritional health education of pregnant women and their developing baby. Previous studies have shown that some midwives self-reported a lack of basic knowledge of nutrition requirements during pregnancy [16,17]. A cross-sectional study that investigated Australian midwives' nutrition knowledge, attitudes, and confidence to provide nutrition education during their undergraduate studies and after registration found that some midwives did not receive evidence-based nutritional health education [16]. It is, therefore, important to investigate the needs of midwives to improve the way in which nutritional health education is provided to pregnant women.

\section{Aim and Objectives}

This paper describes the study protocol for a healthy eating education program during pregnancy. The aim is to design and evaluate a healthy eating education program to enhance midwives' knowledge, understanding, and confidence to support South Australian pregnant women to eat healthily during and after their pregnancy. The study will address the following research objectives through two phases.

Phase 1 will assess midwives' knowledge and level of confidence when providing information on diet and nutrition education for pregnant women and evaluate a healthy eating education program. 
Phase 2 will explore midwives' views on how they provide advice on healthy eating for pregnant women, through an individual interview after they have attended a healthy eating education program.

\section{Methods}

\section{Study Design}

This proposed research study protocol will utilize a sequential explanatory mixed-methods approach guided by a conceptual framework and undertaken in two phases, namely a quantitative phase (Phase 1), followed by a qualitative phase (Phase 2).

\section{Conceptual Framework}

The framework elements in this mixed-methods study are philosophical assumptions (pragmatic knowledge claims), strategies including Sequential Explanatory Mixed-Methods Design, and methods (to describe data collection procedures). These are summarized in Figure 1.

\section{Philosophical Assumptions: Pragmatic Knowledge Claims}

Pragmatism is considered as a worldview, which accepts multiple realities and supports practicality when addressing the research question and it reflects both biased and unbiased perspectives. This pragmatic perspective as a philosophical methodology draws on utilizing "what works," utilizing different aspects of this, setting priority to the value of the research problems and questions, and gathering both objective and subjective data [19].

\section{Strategies Including Sequential Explanatory Mixed-Methods Design}

This proposed research study will use a Sequential Explanatory Mixed-Methods Design according to Creswell [20]. The two phases will gather both quantitative and qualitative data in a sequential manner. The first phase involves collecting and analyzing quantitative descriptive (numeric) data, which gives a general picture and overview of the data related to the research problem. The second phase involves collecting and analyzing qualitative (text) data, which will help explain the general overview and explore the midwives' views in more depth. The interpretation of results is usually undertaken following completion of the second phase. Figure 2, adapted from Jirojwong, Johnson and Welch [21] and Creswell [20], describes this process. Finally, the data obtained from the two phases are integrated [22] to draw a comprehensive and accurate conclusion. The research outcomes using this approach provide a broader, more comprehensive picture of a specific phenomenon compared to using either a quantitative or qualitative method alone [23-25].

\section{Methods of Data Collection}

A semistructured questionnaire will be used in Phase 1 to collect data. The questionnaire was designed and adapted from the literature and previous applied questionnaires $[16,18,26]$ to examine healthy eating and nutrition during pregnancy, as well as problems related to nutrition during pregnancy [17,27-41]. This questionnaire will be used to collect data at 3 time points.

Figure 1. Three framework elements of inquiry for a mixed-methods approach developed from Creswell [22].

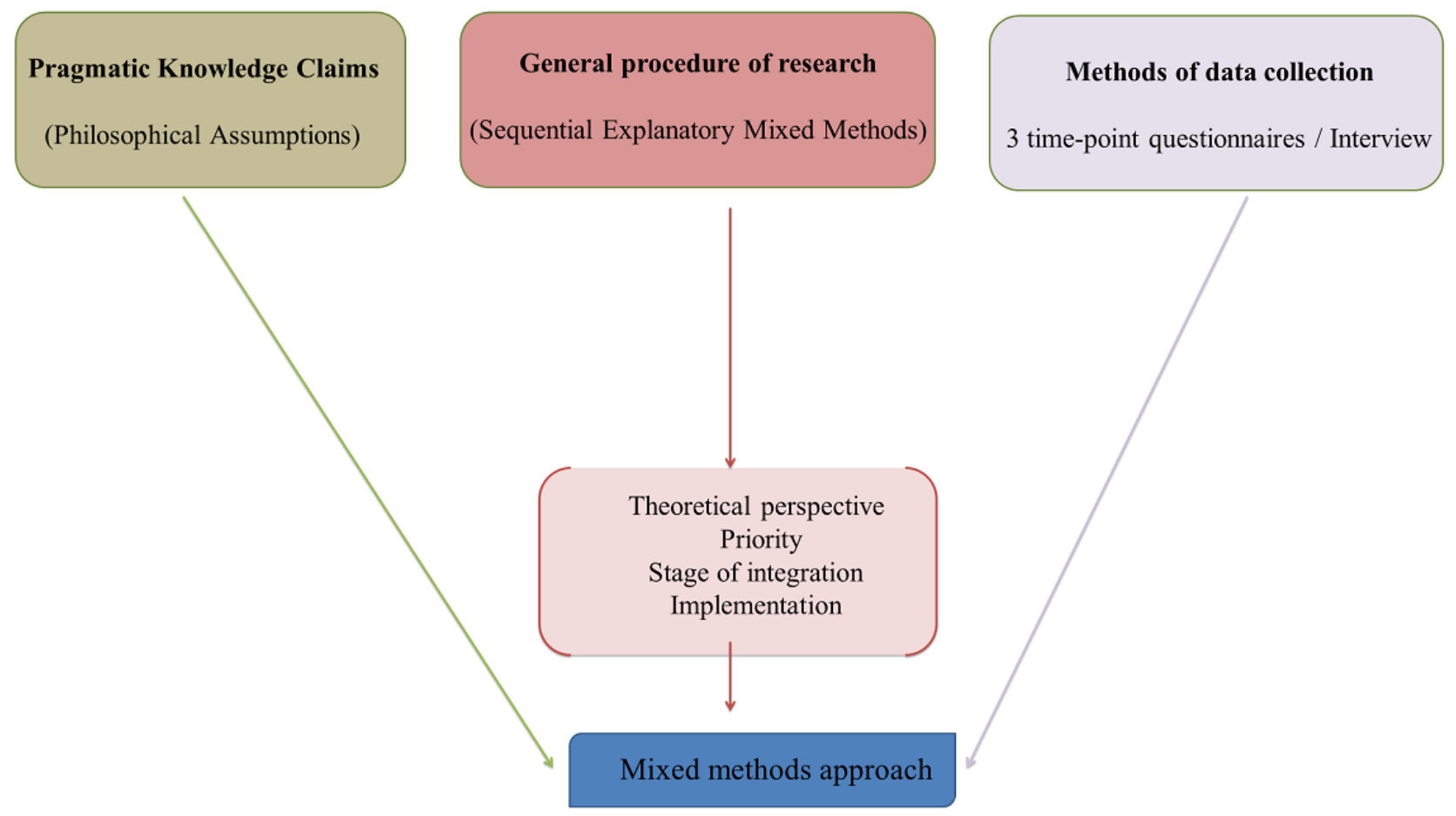


Figure 2. Sequential Explanatory Mixed-Methods Design adapted from Jirojwong, Johnson and Welch [21] and Creswell [20]. ANOVA: analysis of variance; QUAL: qualitative data; QUAN: quantitative data; SPSS: Statistical Package for Social Sciences.

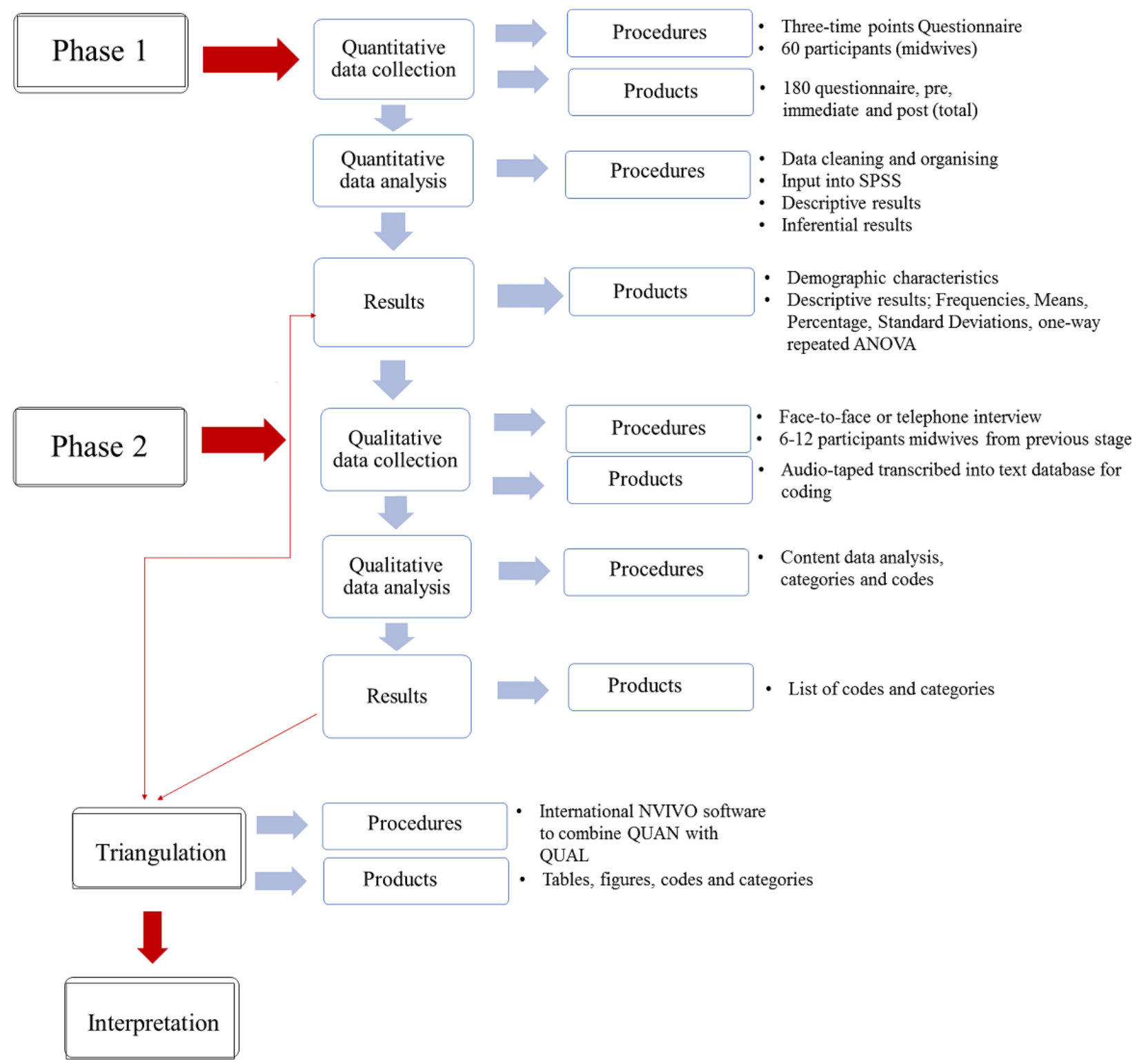

The semistructured, pre-educational questionnaire used in this study included 5 sections asking midwives for their previous nutrition education, level of confidence, nutrition knowledge, an invitation to attend the workshop or webinar, and demographic characteristics based on a previously validated questionnaire (used after seeking permission from the author) [16]. For the immediate- and post-educational questionnaires, midwives will only be asked about their level of confidence and nutrition knowledge.

A pilot of the questionnaire will be undertaken to ascertain validity and reliability of the instrument. Questionnaire validity refers to the extent to which it measures what the questionnaire is intended to measure. Measuring content validity assesses whether the instrument adequately covers all the content with respect to the variables and it is measured by asking experts (face validity testing) their opinion about whether an instrument measures the concept intended [21,42]. The expert panel will be consulted and invited to comment on the questionnaire to give feedback on the instrument (ie, on wording and the order of the questions). Three midwifery experts and 2 dietitians will be invited to review the questionnaire and feedback will be obtained on the clarity of the instrument (questionnaire).

A common method used to estimate the reliability of a measurement is the "test-retest" method, which involves the same test being given to the same participants more than once under the same conditions to evaluate if the responses to the test reflect true variables [42,43]. This questionnaire will undergo a pilot using the "test-retest" method with the aim of assessing whether the same results will be obtained, and therefore its reliability. The pilot will involve three to five midwifery researchers and students who will be invited to participate in the pilot and will not be involved in the main 
study. The midwifery researchers and students involved in the pilot will complete the pre-educational questionnaire twice, once immediately and then again one week later.

Phase 2 of this study will be initiated after the completion of Phase 1. It will consist of a semistructured face-to-face or telephone interview to be scheduled 8 weeks after attendance of the Healthy Eating Education Program. The designed interview schedule will be piloted with three to five midwives or student midwives, and any suggestions or recommendations obtained during the pilot will be incorporated into the final version of the interview schedule.

\section{Phase 1 Methodology: Designing, Facilitating, and Evaluating the Healthy Eating Education Program}

\section{Designing the Workshop or Webinar}

The workshop or webinar will be designed based on results gathered from the systematic review and utilize material from the "EatWell" Assist program [44] and will be designed based on the following criteria: (1) the healthy eating educational program will incorporate evidence-based guidelines and (2) explain the significant nutritional elements including sources, importance, and recommended intake or amount. Furthermore, a workbook will be designed for workshop activities and recommended reading.

\section{Study Website}

The study website will include detailed information about the study. The home page will introduce the study and clearly state its aim and objectives. Other webpages will provide information on how to participate in the study, the ethical considerations related to the study, the research team, and how to contact the primary researcher. Relevant webpages will give prospective participants the option of attending a workshop or webinar, provide online questionnaires to complete, and provide an invitation to attend a follow up face-to-face or telephone interview.

\section{Recruitment}

Midwives residing and employed in South Australia will be invited to participate in the study. An invitation will be sent via the Australian College of Midwives (ACM) and the Australian Nursing and Midwifery Federation (ANMF, South Australian Offices) e-bulletins. A dedicated study website will be designed and utilized with a domain name (http://healthyeating educationformidwives.com/) for midwives to access and participate in the study. An internet advertisement using Google search engine and social media outlets such as Facebook and Twitter will be linked to the study website. A study Facebook page and Twitter account will be set up to increase awareness of the study.

\section{Pre-educational Workshop or webinar Questionnaire to Investigate Midwives' Knowledge and Level of Confidence Regarding Healthy Eating}

A pre-educational healthy eating questionnaire will be undertaken to investigate the midwives pre-existing knowledge, understanding, and level of confidence prior to attending the healthy eating education program.

\section{Settings}

The setting for the participant workshop will be the University of South Australia, School of Nursing and Midwifery, City Campus, Adelaide, or other University of South Australia Campuses, such as Whyalla or Mount Gambier, if this is preferred. For participants who have a preference to attend online or are unable to attend a workshop, an online webinar will be offered.

\section{Implementation of Healthy Eating Education Program}

The healthy eating education program will be delivered by attendance at a workshop or webinar (online virtual classroom).

The first author, SO, will have cofacilitation support to introduce and facilitate a "Healthy Eating in Pregnancy" education program (workshop or webinar) for midwives. The workshop duration will be approximately two hours (including time for refreshment and completing the posttest educational questionnaire). The guide used for the contents covered in the workshop and webinar is described in Textbox 1.

Textbox 1. A guide for content to be covered in the Healthy Eating Education Program workshop or webinar.

- Healthy eating and dietary requirements

- Macronutrients (protein, carbohydrate, fibers, Omega 3 and fats and essential fatty acids), micronutrients (include vitamins A, C, E and B complex and minerals [Se, $\mathrm{Fe}, \mathrm{Ca}, \mathrm{Mg}$, and $\mathrm{Zn}$ ]), folic acid, fish consumption and iodine with pregnancy. The aim of this information is to improve the participant's knowledge about role and sources and how it can enhance health and reduce risk.

- $\quad$ Portion sizes

- $\quad$ Eating myths

- Vegetarians and vegans eating

- Cultural food choices

- Eating habits and behaviors

- $\quad$ Preparing food and hygiene safety

- $\quad$ Physical activity

- Dental care during pregnancy

- $\quad$ Probiotics and prebiotics 
The teaching materials will include a workbook, quiz, brochure, and recommended reading.

\section{Posteducational Workshop and Webinar Questionnaire}

The same pre-educational questionnaire administered to the midwives prior to attendance of the workshop or webinar will be completed again at the end of the workshop or webinar to evaluate the midwives' healthy eating knowledge immediately after the education program. This questionnaire will be provided in a PDF format (for the workshop) and online via the study website for the webinar (virtual classroom), according to the midwives' preference. The researcher and workshop facilitator will remind the midwives that they will be contacted in six to eight weeks after attending the educational program to complete a final questionnaire (using the same pre-educational questionnaire).

\section{Follow-up Educational Workshop or Webinar Questionnaire}

Six to eight weeks after attending the workshop or webinar, the primary researcher will contact the midwives by telephone or email and ask them to complete the final posteducational questionnaire.

\section{Participants or Population}

Midwives residing and employed in South Australia who are members of the ACM and ANMF will be invited to participate in the study. Australian social media groups such as the ACM and ANMF groups on Facebook and Twitter will be provided with a link to the study website.

\section{Sample Size and Power Calculation}

A single-factor, repeated measures design with a sample of 5 subjects, measured at 3 time points, achieves a $91 \%$ power to detect differences among the means using a Geisser-Greenhouse Corrected $F$ Test at a .05 significance level. The SD across subjects at the same time point is assumed to be 25 . The pattern of the covariance matrix is to have all correlations equal with a correlation of .70 between the first and second time point measurements. The SD of the hypothesized means is 17.00 . The expected mean scores over the 3 time points are 50, 90 and 80 .

Based on a power calculation, only a very small number of midwives $(n=5)$ would be required to demonstrate the expected large increase in knowledge and level of confidence as a result of the healthy eating education program (workshop or webinar). However, we will recruit a larger number of participants $(n=60)$ to ensure that we have midwives covering abroad range of age, experiences, and locations.

\section{Data Analysis}

Data from the pre-educational questionnaire will be entered into the Statistical Package for Social Sciences (SPSS) IBM version 25 . Descriptive analysis will be used to examine the midwives' demographic characteristics, such as age, level of education, years of experience, type of maternity services, previous education related to nutrition, and place of practice. The results will be used to describe and summarize the data collected. Data will be presented as frequencies, means, SDs, and percentages. Data related to the level of knowledge and confidence will be analyzed using mixed-effect models to examine the variance in nutritional knowledge and level of confidence over the 3 time points.

Descriptive analysis of questionnaire data will be carried out using an ordinal (1-5 point) Likert scale with the range from 'very confident' (5) to 'not confident at all' (1). Spearman's rank correlations coefficient will be used to evaluate the strength and relationship between two or more variables. Inferential statistics used for making conclusions will compare differences and associations using analysis of variance (ANOVA) tests [25]. Open-ended question responses will be coded as positive or negative, and for content analysis to compare different categories at different time periods [45]. A bio-statistician will be available to assist with the analysis. All questionnaire responses will be numerically coded using a predefined coding scheme.

\section{Phase 2 Methodology: Exploration of Midwives' Views of the Healthy Eating Education Program}

Eight weeks after the Healthy Eating Education Program, a small sample of midwives who participated in the program (12 or less) will be invited to participate in a semi-structured interview either conducted face-to-face or by telephone. An interview schedule will guide the interviews, which will take approximately 30-60 minutes and obtain the participants' views on the healthy eating education they received. The semi-structured interview will help the midwives to express their feelings freely and enable the researcher to encourage midwives to expand more on what is being discussed. However, the researchers will need to remain objective and open to the possibility that the data may be different than expected [25]. The interview guide will be developed from the results of the systematic review (included studies) and findings of Phase 1.

\section{Selection of Participants for Phase 2}

A purposive sample of midwives (approximately 12 or less) will be interviewed 8 weeks after completing the Healthy Eating program. Data from participants' interviews will be recorded and transcribed verbatim. The transcripts will be analyzed on an individual basis until data saturation is reached and recruitment will then cease. The selection of midwives will be based on midwives who work in urban and rural (South Australia) antenatal clinics, Midwifery Group Practices and antenatal parent education classes.

\section{Data Analysis in Qualitative Research}

Content analysis will be used to evaluate the semistructured interview responses. This method is used for identifying, analyzing, and reporting concepts or categories within data. This analysis method minimally organizes and describes data sets in detail. Content analysis as a research strategy is a methodical and systematic method for describing and evaluating phenomena, which allows for testing theoretical issues to establish understanding of data and comprehension of information $[46,47]$. The aim of utilizing content analysis will be to achieve a broad description of midwives' views after receiving the healthy eating education. 


\section{Content Analysis Process}

Interview content analysis will be based on the Conventional content analysis approach which will assist to gain more insights on healthy eating in pregnancy education for midwives after they have received the training [48]. The 3-phase framework for content analysis as described by Elo and Kyngas [45] will be utilized as described below.

\section{Preparation Phase}

This phase commences with selecting the unit of analysis, this unit can be a word or a theme, relevant to the research question. Using the manifest content where the researcher describes what information was actually said (ie, uses the same words and describes the visible and what is said in the text) [49].

\section{Organizing Phase}

After making sense of the data, an inductive approach will be used starting from open coding for whole reading of the dataset, creating grouping and categories, then abstraction to formulate a general description of the research topic through generating main categories, generic categories, and subcategories from analyzed data.

\section{Reporting the Analyzing Process and the Results}

A final report will be written to provide brief, classified, logical, varied, realistic, and interesting conclusions from the story obtained from the data obtained, within, and across, all codes and categories.

\section{Results}

\section{Triangulation of Quantitative and Qualitative Data Findings}

The sequential explanatory design enables the reporting of participants' cases as one continued story starting from the pre-educational questionnaire, then the healthy eating education workshop or webinar, continued to the participants attending an interview based on the findings from the systematic review and finally to make general conclusions about the participants' knowledge, level of confidence views, and understanding their role in healthy eating education. According to the conceptual framework (Figure 1) and the sequential explanatory design discussed in Figure 2, an initial data analysis will be undertaken when completing the systematic review. Moreover, this analysis will continue during Phase 1 (quantitative data extracted from questionnaires with closed- and open-ended questions). The results of the systematic review and the quantitative phase will help to develop a guide for the semi-structured, in-depth interviews with midwives. Semi-structured interview data will be analyzed using content analysis, and the results outcome from this phase will be integrated together with the previous analysis to obtain a broader and more accurate picture describing midwives' nutritional knowledge and confidence.

\section{Ethical Considerations}

Ethics approval has been granted from the Human Research Ethics Committee at the University of South Australia. Approval has been given to advertise the study via the ACM and the ANMF professional bodies to invite midwives to participate in the study. The program will also be recognized by the ACM for continual professional development points. The link to the study website will be included. The study website will also be linked to the Australian Midwives social media groups such as the ACM and ANMF groups on Facebook and Twitter. To maintain confidentiality, a unique identifying number (participant ID) will be assigned to the data collected for each participant. Participation in the study is voluntary and contact details of the research team and a person who is responsible for receiving any complaints will be provided on the study website.

Participants will be able to access a participant information sheet that will outline the purpose of the study, the phases and its importance, via the study website. Participation is voluntary, and midwives can withdraw at any time without influencing their status now or in the future. The participants will be informed about the upcoming workshops and interviews if they wish to participate, and interviews will be audiotaped for further analysis. The transcripts and tape will be securely stored in a locked cabinet in a locked room and only the research team will have access to it.

The results will be nonidentifiable, and findings will be published in journals and at conferences. Participants will be able to access a summary of the findings from the study website. Anticipated benefits of the study include an increase in the level of knowledge for midwives regarding nutritional healthy eating education. Midwives who participate in the health education program (workshop or webinar) will be given two points of Continuing Professional Development on completing the education program through the ACM.

\section{Discussion}

\section{Study Rationale}

It is anticipated that there will be several expected benefits from undertaking and participating in this proposed research. For example, midwives who reside in South Australia will have an opportunity to attend a healthy eating education program (either as a workshop facilitated on campus at the University of South Australia and also accessible as an internet-based webinar) which will be freely available to access and be validated through this research study. This healthy eating educational opportunity has the potential to enhance the knowledge and confidence of midwives who reside in South Australia. It is envisaged that participation in this study will improve midwives' awareness and knowledge of evidence-based guidelines regarding healthy eating in pregnancy. In addition, midwives' confidence may increase to enable them to provide additional nutritional healthy eating education and support for pregnant women. Should this program increase the midwives' knowledge and confidence to educate women, this may improve maternal and fetal outcomes.

\section{Conclusion}

The preliminary results of the on-going systematic review suggest that there is clear justification to undertake this mixed-methods study to investigate and explore midwives' knowledge, understanding and confidence to support pregnant women to eat healthily. Findings and conclusions from this systematic review has provided some guidance for the design 
and development of this protocol. The questionnaire has been designed to be used at 3 time points during the first phase of this research study. A follow up second phase will provide an opportunity to gain a more in-depth understanding of midwives' views after they have received the healthy eating education program. The results from both phases (quantitative and qualitative) data sources will help to draw conclusions to address the research topic.

\section{Acknowledgments}

The PhD candidate, SMEO, acknowledges Cultural Affairs and Mission Sector, Ministry of Higher Education, Egypt, for the fully funded scholarship to undertake a PhD study at the University of South Australia. The Egyptian sponsor did not play any roles or impose any restrictions in the process.

\section{Authors' Contributions}

All authors listed have contributed sufficiently to the development of ideas and the writing of the manuscript.

\section{Conflicts of Interest}

None declared.

\section{Multimedia Appendix 1}

Peer-reviewer report.

\section{[PDF File (Adobe PDF File), 335KB-Multimedia Appendix 1]}

\section{References}

1. Downs DS, Chasan-Taber L, Evenson KR, Leiferman J, Yeo S. Physical activity and pregnancy: past and present evidence and future recommendations. Res Q Exerc Sport 2012 Dec;83(4):485-502 [FREE Full text] [doi:

10.1080/02701367.2012.10599138] [Medline: 23367811]

2. Barger MK. Maternal nutrition and perinatal outcomes. J Midwifery Womens Health 2010;55(6):502-511 [FREE Full text] [doi: 10.1016/j.jmwh.2010.02.017] [Medline: 20974412]

3. Han Z, Mulla S, Beyene J, Liao G, McDonald SD, Knowledge Synthesis Group. Maternal underweight and the risk of preterm birth and low birth weight: a systematic review and meta-analyses. Int J Epidemiol 2011 Feb;40(1):65-101. [doi: 10.1093/ije/dyq195] [Medline: 21097954]

4. Jones KL. The effects of alcohol on fetal development. Birth Defects Res C Embryo Today 2011 Mar;93(1):3-11. [doi: 10.1002/bdrc.20200] [Medline: 21425437]

5. Cnattingius S, Villamor E, Johansson S, Edstedt Bonamy AK, Persson M, Wikström AK, et al. Maternal obesity and risk of preterm delivery. JAMA 2013;309(22):2362-2370. [doi: 10.1001/jama.2013.6295] [Medline: 23757084]

6. Phelan S. Smoking cessation in pregnancy. Obstet Gynecol Clin North Am 2014;41(2):255-266. [doi: 10.1016/j.ogc.2014.02.007] [Medline: 24845489]

7. Vieau D. Perinatal nutritional programming of health and metabolic adult disease. World J Diabetes 2011 Sep 15;2(9):133-136 [FREE Full text] [doi: 10.4239/wjd.v2.i9.133] [Medline: 21954417]

8. Balbus JM, Barouki R, Birnbaum LS, Etzel RA, Gluckman PD, Grandjean P, et al. Early-life prevention of non-communicable diseases. The Lancet 2013 Jan;381(9860):3-4. [doi: 10.1016/s0140-6736(12)61609-2]

9. Banderali G, Martelli A, Landi M, Moretti F, Betti F, Radaelli G, et al. Short and long term health effects of parental tobacco smoking during pregnancy and lactation: a descriptive review. J Transl Med 2015 Oct 15;13:327 [FREE Full text] [doi: 10.1186/s12967-015-0690-y] [Medline: 26472248]

10. Kapur A. Links between maternal health and NCDs. Best Pract Res Clin Obstet Gynaecol 2015 Jan;29(1):32-42. [doi: 10.1016/j.bpobgyn.2014.04.016] [Medline: 25199858]

11. Baron R, Heesterbeek Q, Manniën J, Hutton EK, Brug J, Westerman MJ. Exploring health education with midwives, as perceived by pregnant women in primary care: A qualitative study in the Netherlands. Midwifery 2017 Mar;46:37-44. [doi: 10.1016/j.midw.2017.01.012] [Medline: 28161688]

12. Edvardsson K, Ivarsson A, Eurenius E, Garvare R, Nyström ME, Small R, et al. Giving offspring a healthy start: parents' experiences of health promotion and lifestyle change during pregnancy and early parenthood. BMC Public Health 2011 Dec 15;11:936 [FREE Full text] [doi: 10.1186/1471-2458-11-936] [Medline: 22171644]

13. Guelinckx I, Devlieger R, Mullie P, Vansant G. Effect of lifestyle intervention on dietary habits, physical activity, and gestational weight gain in obese pregnant women: a randomized controlled trial. Am J Clin Nutr 2010 Feb;91(2):373-380. [doi: 10.3945/ajcn.2009.28166] [Medline: 19955397]

14. McNeill J, Doran J, Lynn F, Anderson G, Alderdice F. Public health education for midwives and midwifery students: a mixed methods study. BMC Pregnancy Childbirth 2012 Dec 07;12:142 [FREE Full text] [doi: 10.1186/1471-2393-12-142] [Medline: 23216855] 
15. Othman SME, Jayasekara R, Steen M, Fleet J. A systematic review protocol for exploring the effectiveness of healthy eating education programmes for improving midwives level of knowledge and confidence in promoting healthy eating in pregnant women. Evidence Based Midwifery 2018 (in print).

16. Arrish J, Yeatman H, Williamson M. Australian midwives and provision of nutrition education during pregnancy: A cross sectional survey of nutrition knowledge, attitudes, and confidence. Women Birth 2016 Oct;29(5):455-464. [doi:

10.1016/j.wombi.2016.03.001] [Medline: 27020228]

17. Basu A, Kennedy L, Tocque K, Jones S. Eating for 1, Healthy and Active for 2; feasibility of delivering novel, compact training for midwives to build knowledge and confidence in giving nutrition, physical activity and weight management advice during pregnancy. BMC Pregnancy Childbirth 2014 Jul 04;14:218 [FREE Full text] [doi: 10.1186/1471-2393-14-218] [Medline: 24996422]

18. Pan S, Dixon L, Paterson H, Campbell N. New Zealand LMC midwives? approaches to discussing nutrition, activity and weight gain during pregnancy. NZCOM Journal 2014;50(4):24-29 [FREE Full text] [doi: 10.12784/nzcomjn150.2014.4.24-29]

19. Morgan DL. Paradigms Lost and Pragmatism Regained, Methodological implications of combining qualitative and quantitative methods. Journal of Mixed Methods Research 2007;1(1):48-76. [doi: 10.1177/2345678906292462]

20. Creswell J. A Concise Introduction to Mixed Methods Research. Ltd. , Los Angeles: SAGE; 2015.

21. Jirojwong S, Johnson M, Welch A. Research Methods in Nursing and Midwifery: Pathways to Evidence-based Practice. 2nd ed. Sydney: Oxford University Press; 2014.

22. Creswell J. Research design: Qualitative, quantitative and mixed methods approaches. 2nd ed. Thousand Oaks, California: Sage; 2003.

23. Leedy P, Ormrod J. Practical Research ( Planning and Design ). 9th ed. USA: Pearson Education International; 2010.

24. Creswell J. Research Design, qualitative, Quantitaive, and Mixed Methods Approaches. 4th ed. Thousand Oaks, California: SAGE; 2014.

25. Mcintosh-Scott A, Mason T, Mason-Whitehead E, Coyle D. Key Concepts in Nursing and Healthcare Research. UK: SAGE; 2013.

26. Oza-Frank R, Ko JY, Wapner A, Rodgers L, Bouchard JM, Conrey EJ. Improving care for women with a history of gestational diabetes: a provider perspective. Matern Child Health J 2014 Sep;18(7):1683-1690. [doi: 10.1007/s10995-013-1410-y] [Medline: 24343308]

27. Greenberg JA, Bell SJ, Ausdal WV. Omega-3 Fatty Acid supplementation during pregnancy. Rev Obstet Gynecol 2008;1(4):162-169. [Medline: 19173020]

28. Malek L, Umberger W, Makrides M, Zhou SJ. Poor adherence to folic acid and iodine supplement recommendations in preconception and pregnancy: a cross-sectional analysis. Aust N Z J Public Health 2016 Oct;40(5):424-429. [doi: 10.1111/1753-6405.12552] [Medline: 27523027]

29. Malek L, Umberger W, Makrides M, Zhou SJ. Adherence to the Australian dietary guidelines during pregnancy: evidence from a national study. Public Health Nutr 2016 May;19(7):1155-1163. [doi: 10.1017/S1368980015002232] [Medline: 26228526]

30. Martin JC, Savige GS, Mitchell EKL. Health knowledge and iodine intake in pregnancy. Aust N Z J Obstet Gynaecol 2014 Aug;54(4):312-316. [doi: 10.1111/ajo.12201] [Medline: 24628031]

31. Dodd JM, Turnbull D, McPhee AJ, Deussen AR, Grivell RM, Yelland LN, et al. Antenatal lifestyle advice for women who are overweight or obese: LIMIT randomised trial. BMJ 2014 Feb 10;348(feb10 3):g1285-g1285. [doi: 10.1136/bmj.g1285]

32. American College of Obstetricians and Gynaecologists, Committee on Obstetric Practice. 2013. Weight gain during pregnancy URL: https://www.acog.org/Clinical-Guidance-and-Publications/Committee-Opinions/

Committee-on-Obstetric-Practice/Weight-Gain-During-Pregnancy [accessed 2018-05-09] [WebCite Cache ID 6zHH6nYyt]

33. Eat for Health. 2015. Australian Dietary Guidelines, Advice about the amount and kinds of foods that we need to eat for health and wellbeing URL: https://www.eatforhealth.gov.au [accessed 2018-05-09] [WebCite Cache ID 6zHHESiLf]

34. Division of Reproductive Health. 2016. Weight gain during pregnancy, Center for Disease Control andprevention URL: https://www.cdc.gov/reproductivehealth/maternalinfanthealth/pregnancy-weight-gain.htm [accessed 2018-05-09] [WebCite Cache ID 6zHHTfJN0]

35. Better Health Channel. 2017. Healthy Pregnancy, Victoria State Government URL: https://www.betterhealth.vic.gov.au/ healthyliving/healthy-pregnancy

36. Nutrition Australia. 2017. Australian Dietary Guidelines: Recommended Daily intakes URL: http://www.nutritionaustralia.org/ $\underline{\text { national/resource/australian-dietary-guidelines-recommended-daily-intakes [accessed 2018-05-09] [WebCite Cache ID }}$ 6zHIK4XNF]

37. Institute of Medicine. Washington DC: National Academy Press; 2009. Nutrition During Pregnancy, National Academy of Sciences, Institute of Medicine, Food and Nutrition Board, Committee on Nutritional Status During Pregnancy and Lactation, Subcommittee on Dietary Intake and Nutrient Supplements During Pregnancy, Subcommittee on Nutritional Status and Weight Gain During Pregnancy URL: http://www.nationalacademies.org/hmd/ /media/Files/Report\%20Files/ 2009/Weight-Gain-During-Pregnancy-Reexamining-the-Guidelines/ Report\%20Brief\%20-\%20Weight\%20Gain\%20During\%20Pregnancy.pdf [accessed 2018-05-09] [WebCite Cache ID 6zHIyh1vm] 
38. National Health and Medical Research Council. 2013. Australian Dietary Guidelines, Canberra, National Health and Medical Research Council URL: https://www.nhmrc.gov.au/guidelines-publications/n55 [accessed 2018-05-09] [WebCite Cache ID 6zHJ5RBIg]

39. Department of Health. 2011. Weight and body mass index, Australian government URL: http://www.health.gov.au/internet/ publications/publishing.nsf/Content/clinical-practice-guidelines-ac-mod1 part-b clinical-assessments+ weight-bmi [accessed 2018-05-09] [WebCite Cache ID 6zHJDEI4w]

40. Gardiner P, Jarrett K, Filippelli A, Pecci C, Mauch M, Jack B. Herb use, vitamin use, and diet in low-income, postpartum women. J Midwifery and Womens Health 2013 Apr;58(2):150-157 [FREE Full text] [doi: 10.1111/j.1542-2011.2012.00240.x] [Medline: 23590486]

41. Williamson C, Lean MEJ, Combet E. Dietary iodine: awareness, knowledge and current practice among midwives. In: Proc. Nutr. Soc. 2012 Oct 19 Presented at: Proceedings of the nutrition society; 16-19 July; Cambridge. [doi: 10.1017/S0029665112001991]

42. Heale R, Twycross A. Validity and reliability in quantitative studies. Evid Based Nurs 2015 Jul;18(3):66-67. [doi: 10.1136/eb-2015-102129] [Medline: 25979629]

43. Steen M, Roberts T. The Handbook of Midwifery Research Vol. 1st ed. UK: Wiley-Blackwell; 2011.

44. Steen M, Mottershead R, Idriss J, Parletta N, Aftab B, Kumardhas V. Diet and eating habits of expectant parents and families in Ras Al Khaimah, Emirates: an exploratory study. Evidence Based Midwifery 2017;15(2):a-53.

45. Elo S, Kyngäs H. The qualitative content analysis process. J Adv Nurs 2008 Apr;62(1):107-115. [doi: 10.1111/j.1365-2648.2007.04569.x] [Medline: 18352969$]$

46. Sandelowski M. Qualitative analysis: What it is and how to begin. Res. Nurs. Health 1995 Aug;18(4):371-375. [doi: 10.1002/nur.4770180411] [Medline: 7624531]

47. Krippendorff K. Content Analysis An Introduction to Its Methodology. California: Thousand Oaks; 2004.

48. Hsieh H, Shannon SE. Three approaches to qualitative content analysis. Qual Health Res 2005 Nov;15(9):1277-1288. [doi: 10.1177/1049732305276687] [Medline: 16204405]

49. Bengtsson M. How to plan and perform a qualitative study using content analysis. NursingPlus Open 2016;2(Supplement C):8-14. [doi: 10.1016/j.npls.2016.01.001]

\section{Abbreviations \\ ACM: Australian College of Midwives \\ ANMF: Australian Nursing and Midwifery Federation \\ ANOVA: analysis of variance \\ SPSS: Statistical Package for Social Sciences}

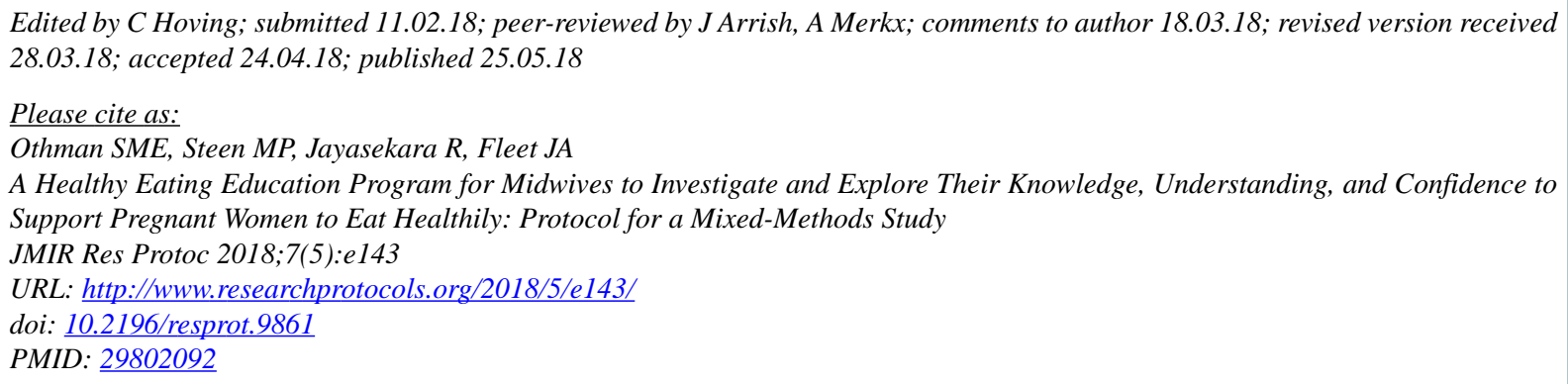

(CShwikar Mahmoud Etman Othman, Mary P Steen, Rasika Jayasekara, Julie-Anne Fleet. Originally published in JMIR Research Protocols (http://www.researchprotocols.org), 25.05.2018. This is an open-access article distributed under the terms of the Creative Commons Attribution License (https://creativecommons.org/licenses/by/4.0/), which permits unrestricted use, distribution, and reproduction in any medium, provided the original work, first published in JMIR Research Protocols, is properly cited. The complete bibliographic information, a link to the original publication on http://www.researchprotocols.org, as well as this copyright and license information must be included. 